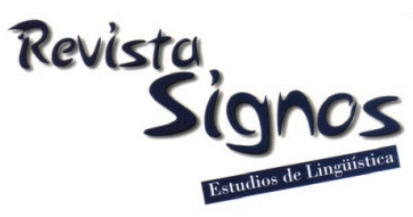

\title{
Relación entre conciencia morfológica y destreza lectora: Un estudio con niños hispanohablantes
}

\section{Relationship between morphological awareness and reading skills: A study with Spanish speaking children}

\section{Miguel Lázaro}

UNIVERSIDAD COMPLUTENSE DE MADRID ESPAÑA

mlazaro@ucm.es

\section{Ainoa Escalonilla}

UNIVERSIDAD COMPLUTENSE DE MADRID ESPAÑA

ainoaesc@ucm.es

\author{
Trinidad Ruiz Gallego-Largo \\ UNIVERSIDAD COMPLUTENSE DE MADRID \\ EsPaña \\ truiz@psi.ucm.es
}

Teresa Simón

UNIVERSIDAD COMPLUTENSE DE MADRID ESPAÑA

tsimon@ucm.es

Recibido: 21-V-2019 / Aceptado: 09-VI-2020

DOI: $10.4067 /$ S0718-09342021000100032

\section{Resumen}

En este trabajo se indaga en la relación entre conciencia morfológica y destreza lectora en niños hablantes nativos de español de tercero y cuarto de primaria. A los participantes se les administraron pruebas de: conciencia fonológica, nivel de vocabulario, cociente intelectual, velocidad lectora, destreza lectora y una prueba diseñada ad hoc de conciencia morfológica. Esta tarea consta de 8 subtareas que comprenden tanto la morfología derivativa como la flexiva. Los resultados muestran que para la flexión no existe un efecto evolutivo y sí un efecto techo, mientras que para la derivación sí se observa un efecto evolutivo. Por otro lado los resultados de la prueba de conciencia morfológica correlacionan significativamente con los de comprensión lectora, lo que avala la relevancia de esta variable en la comprensión de textos. Igualmente los resultados en conciencia morfológica correlacionan con el nivel de vocabulario, lo que demuestra la naturaleza léxica de esta variable. Estos resultados son explicados a la luz de las investigaciones previas realizadas en diversas lenguas, haciendo hincapié en las obtenidas en español.

Palabras Clave: Conciencia fonológica, conciencia morfológica, destreza lectora, inteligencia, nivel de vocabulario. 


\begin{abstract}
This study focuses on the realationship between morphological awareness and reading skills in third and fourth graders. Participants were evaluated in phonological awareness, vocabulary level, IQ, reading speed, reading comprehension and in a morphological processing task. This task is formed by 8 different subtasks both on inflectional and derivational morphology. The results show a lack of a developmental effect in the case of inflectional morphology but a significant effect for derivational morphology. On the other hand, the results on the morphological awareness task correlate significantly with the reading comprehension, therefore supporting the relevance of this variable for reading. The results on the morphological awareness task also correlate with the vocabulary level, thus showing the lexical nature of this variable. The overall results are discussed in the light of previous research carried out in different languages including Spanish.
\end{abstract}

Key Words: Sentence comprehension, gender stereotypes, eye movements, syntactic parsing models.

\title{
INTRODUCCIÓN
}

La conciencia morfológica se refiere al conocimiento que una persona tiene sobre la composición interna de las palabras en términos morfológicos, es decir, al conocimiento acerca de la formación morfológica de las palabras. Dicho de otro modo, la conciencia morfológica consiste en la capacidad de reconocer, comprender y usar morfemas para entender o crear palabras (Apel, 2014 para una completa discusión acerca de la definición de conciencia morfológica; Carlisle, 2000). Así, por ejemplo, la capacidad de relacionar las palabras 'cochera' y 'coche' se debe a nuestra conciencia morfológica así como que, si proponemos la pseudopalabra 'suelista', un hablante nativo de español pueda razonablemente proponer como definición 'persona que pone suelos en las casas o edificios'.

Respecto de la morfología existe un hecho fundamental que permite inmediatamente comprender la posible importancia de la conciencia morfológica. Se trata de que la mayor parte de las palabras de nuestra lengua está compuesta por palabras derivadas, es decir, las palabras monomorfémicas como 'cabeza', 'playa' o ‘teatro' no son las más numerosas, sino que, por el contrario, palabras polimorfémicas como 'cabecero', 'playera' o 'teatral' son más numerosas (Booig, 2002, para un estudio específico del holandés y Rey-Debove, 1984, para el francés). Así pues, los aspectos relacionados con el posible papel de la conciencia morfológica no se limitan a un debate más o menos intelectual alejado de la realidad de los hablantes, sino que, al contrario, su influencia, de demostrarse, podría ser enorme habida cuenta de su continua presencia en el lenguaje. 


\section{Marco teórico}

Uno de los primeros estudios experimentales sobre el desarrollo del conocimiento morfológico fue el desarrollado por Berko (1958). Esta autora investigó en niños de lengua inglesa de distintas edades la habilidad de aplicar reglas morfológicas, flexivas y derivativas, no solo con palabras reales, sino también con pseudopalabras. El trabajo de Berko se denomina Wug test precisamente por ser ésta una de las pseudopalabras empleadas. Así, se les decía a los niños, 'esto es un wug y estos son dos...' mientras se les enseñaban unas láminas y se esperaba su respuesta. Esta tarea ha sido empleada posteriormente innumerables veces con distintos desarrollos. Por ejemplo, Selvy (1972) realizó esta tarea no solo para evaluar la generación de plurales sino también la de comparativos, superlativos etc. en niños de entre 4 y doce años en lengua también inglesa. En este trabajo de Selvy (1972) se remarcó un aspecto que es fundamental para los propósitos de este trabajo, y es que a la edad de 12 años se alcanzaban ya efectos techo en la mayoría de los aspectos morfológicos estudiados. En el caso del español, Pérez-Pereira (1989) aplicó el test Wug con niños de entre 3 y 6 años. Además de encontrar diferencias entre los alomorfos (menos errores en el caso de la aplicación del alomorfo -s que del-es), observó que a la edad de 6 años los niños ya no cometían errores con el alomorfo $-\mathrm{s}$ y solo una tasa mínima en el caso del alomorfo -es, es decir, que a los 6 años parecía estar prácticamente adquirido la formación de plurales. A esta misma conclusión llegaron Lázaro, Nieva, Moraleda y Garayzábal (2013) tanto con niños con desarrollo típico como con niños con síndrome de Down. Así pues parece que la flexión nominal está completamente adquirida de forma relativamente temprana, y más tempranamente aun empleada sistemáticamente, pues a la edad de 2,6 años existen indicios de este uso recurrente (Marrero \& Aguirre 2003). La idea de que el conocimiento morfosintáctico no se desarrolla sino tempranamente, hasta los tres o cuatro años, tiene su raíz en un enfoque muy innatista del proceso de adquisición (López-Ornat, 2011) que creemos ha sido ya superado. Así, sabemos por ejemplo que los aspectos supraoracionales como la generación del discurso se desarrolla en edades notablemente más tardías. No obstante, el conocimiento morfosintáctico comienza de un modo muy temprano y termina a nivel oracional de un modo igualmente temprano. Subrayamos, por su importancia para este trabajo, que la evidencia experimental muestra que el curso evolutivo de la adquisición del conocimiento morfológico flexivo es distinto al derivativo dado que este último se desarrolla hasta una edad notablemente más tardía (Deacon \& Bryant, 2005; González, Rodríguez, Gázquez, González \& Álvarez, 2011; Mahony, Singson \& Mann, 2000; Tyler \& Nagy, 1989).

La diferencia entre el conocimiento de la morfología flexiva y derivativa no radica únicamente en los cursos temporales del aprendizaje de ambas, sino que resulta más importante aún la distinta naturaleza de ambas 'morfologías'. En efecto, pese a recibir un mismo nombre genérico de 'morfología', la flexiva tiene una función puramente 
gramatical que no coincide con la derivativa en modo alguno, cuya naturaleza es en mayor medida léxica. La flexión es ante todo la realización de la sintaxis a nivel de palabra (Aronoff \& Fudeman, 2005; Stump, 1998), es decir, que expresa la dependencia de las palabras entre sí codificando relaciones sintácticas, mientras que la derivación (en cualquiera de sus formas, prefijación, sufijación y composición) constituye el procedimiento natural para generar nuevas palabras (Varela, 1993). En último término la distinción entre flexión y derivación no es sino la distinción entre las propiedades morfosintácticas y léxico-semánticas de las lenguas. Se trata de procesos lingüística y cognitivamente tan distintos que, de hecho, dan lugar a un universal lingüístico. Así, Moreno (1997) observa que la composición es un procedimiento universal de formación de nuevas palabras. La afijación (prefijación y sufijación) es un procedimiento prácticamente universal, mientras que la flexión es característica de algunas familias lingüísticas, pero que está ausente en otras muchas familias. En términos formales puede definirse este universal del modo siguiente (Greenberg, 1963):

\section{Composición > Afijación > Flexión}

Por lo tanto, todas las lenguas conocen recursos para generar nuevas palabras a partir de otras, principalmente la composición por su relativa simplicidad cognitiva (Spencer, 1998), pero no ocurre lo mismo con la jerarquización de las mismas en el discurso porque hay otros recursos que pueden sustituir su función como, por ejemplo, a través de un orden marcado de palabras en la oración (como ocurre en lenguas como el chino por ejemplo, Moreno (2003). A nuestro juicio, el conocimiento que se tenga de cómo se generan nuevas palabras no tiene por qué necesariamente relacionarse con el modo en que se jerarquizan dichas palabras en una oración, o dicho de otro modo, el estudio de la morfosintaxis no debe confundirse con el estudio a nivel léxico-semántico. Así pues defendemos que los estudios sobre conciencia morfológica no deben integrar los resultados que de ambos tipos de conocimiento morfológico tengan los hablantes, sino que, más bien, resulta prudente estudiar ambos por separado. En este trabajo nos centraremos en la morfología derivativa, si bien introduciremos la flexiva para tratar de demostrar el distinto curso temporal del que ya se ha hablado anteriormente.

Con respecto a la conciencia morfológica derivativa existen numerosos trabajos, si bien en lenguas que no son la española, donde la evidencia es mucho menor. Mann y Singson (2003), por ejemplo, encontraron resultados muy relevantes. Estos autores indican que la conciencia morfológica experimenta un importante incremento entre tercero y sexto de primaria, es decir, entre los 8 y 9 años y los 11 y 12 respectivamente y que este incremento se relaciona con la habilidad lectora. En su estudio desarrollan varios experimentos en los que observan que las habilidades lectoras son explicadas no solo por el nivel de vocabulario y de conciencia fonológica, que son variables ya muy 
exploradas en la investigación psicolingüística, sino también por la conciencia morfológica. Además, en sus resultados mostraron que la conciencia morfológica era la variable que mayor porcentaje de la varianza explicaba. Por ello, concluyeron que la conciencia morfológica no solo contribuye a la habilidad lectora, sino que contribuye más que otras variables más conocidas experimentalmente.

Por su parte, McBride-Chang, Tardif, Cho, Shu, Fletcher, Stokes, Wong y Leung (2008) realizaron un estudio con más de 600 niños hablantes de chino mandarín, cantonés y coreano en el que se puso en juego la habilidad para crear compuestos (procedimiento para generar nuevas palabras muy productivo en estas lenguas). En sus resultados se observó un papel importante tanto para el nivel de vocabulario como para las conciencias fonológica y morfológica, si bien destacaron en sus conclusiones que existe una relación bidireccional entre conciencia morfológica y adquisición de vocabulario por la cual no solo la conciencia morfológica contribuye a la adquisición de nuevo vocabulario, sino que el nivel de vocabulario contribuye al desarrollo de la conciencia morfológica. Finalmente concluyen estos autores que el nivel de conciencia morfológica, aun poco estudiado a su juicio, es un potente predictor del nivel de vocabulario en lenguas como las mencionadas en las que la composición es un procedimiento muy productivo (ver también McBride-Chang, Wagner, Muse, Chow, y Shu, 2005, similares conclusiones en un estudio con lengua inglesa).

Es importante destacar que las investigaciones sobre el papel de la conciencia morfológica derivativa no solo se han llevado a cabo con tareas en las que se leen palabras aisladas, es decir, en investigaciones que propiamente relacionan los niveles de conciencia morfológica con habilidades de lectura de palabras. La evidencia con que contamos también se refiere a la relación entre las habilidades lectoras a nivel de texto -comprensión lectora- y el conocimiento morfológico (Carlisle, 2000; Kieffer \& Lesaux, 2008; Verhoeven \& Perfetti, 2003). Por ejemplo, Carlisle y Fleming (2003) realizaron un estudio longitudinal en el que evaluaron en un primer momento a grupos de niños en pruebas de conciencia morfológica y, dos años después, les administraron pruebas de comprensión y destreza lectora. Estos autores observaron que la habilidad a la hora de realizar las tareas propuestas al principio del estudio correlacionaba positiva y significativamente con las capacidades de lectura comprensiva de los niños dos años después. Carlisle y Fleming (2003) observaron, pues, que existe una estrecha relación entre conciencia morfológica y destreza lectora por la cual los niños con mejores resultados en las tareas de conciencia morfológica muestran una mayor competencia lectora dos años después.

En el caso del español, como antes se mencionó, existen investigaciones sobre conciencia morfológica, incluso trabajos teóricos sobre ella (Mínguez, 2018), si bien son poco numerosas y, por tanto, con razonables lagunas que ir cubriendo con nuevos estudios. Es importante porque en el área del lenguaje, y de la morfología en particular, los resultados para unas lenguas no representan necesariamente lo que 
suceda en otras. Así, algunos resultados para el chino pueden no suponerse para el inglés (McBride-Chang et al., 2005) o los del inglés para el español, por ejemplo, pues se trata de dos lenguas muy distintas en términos morfológicos. Es preciso, por tanto, desarrollar la investigación en esta última lengua.

En español, Vega, Torres y del Campo (2016) evaluaron en 36 niños chilenos su nivel de conciencia morfológica y sus habilidades lectoras. Las pruebas de conciencia morfológica se realizaron ad hoc para el estudio, finalmente haciendo uso de cuatro tareas de morfología derivativa. Los resultados mostraron una correlación positiva y significativa entre los niveles de conciencia morfológica y la habilidad lectora, evaluada ésta a través de un test estandarizado. Aunque la medición de la conciencia morfológica a través de pruebas derivativas se adecúa perfectamente a nuestra argumentación y justificación del presente estudio, el número de participantes relativamente escaso y sobre todo la falta de otras medidas como el nivel de vocabulario, por ejemplo, hace que esos resultados deban ser considerados con cautela porque no permiten conocer la contribución de la conciencia morfológica de forma independiente a otras variables relevantes en la destreza lectora.

Por su parte, González et al. (2011) realizaron un estudio con 50 estudiantes de entre 5 y 7 años. Se evaluó a estos niños en dos ocasiones a través del test IECME para conocer los niveles de conciencia morfológica en un primer momento y también en la segunda medición. Este test ofrece un sumatorio de distintas pruebas morfológicas en donde la flexión y la derivación no se distinguen y, según los propios autores, pese a los buenos resultados psicométricos obtenidos inicialmente, serán necesarias ciertas modificaciones para su final desarrollo. En sus resultados se observa una progresión según la cual la pluralidad y el género se desarrollan antes que la morfología derivativa, en consonancia con lo anteriormente expuesto sobre los cursos evolutivos flexivo y derivativo. Además, observan que las puntuaciones en conciencia morfológica aumentan con la edad, aunque la falta de mediciones sobre otras variables como la conciencia fonológica o el nivel de vocabulario vuelven de nuevo a limitar la posible interpretación del patrón evolutivo observado (García \& González (2006) para un estudio muy similar en lengua escrita).

Rueda y Medina (2018) realizaron, por su parte, un estudio en el que evaluaron los niveles de conciencia morfológica en 54 niños con diagnóstico de trastorno lector. Realizaron pruebas tanto de conciencia morfológica, como de conciencia fonológica, nivel de vocabulario y lectura en voz alta y deletreo. Sus resultados correlacionales mostraron que cuanto mayor era la conciencia morfológica menos eran los errores de lectura y mayor la destreza en el deletreo. Los mismos resultados se observaron para el nivel de vocabulario, es decir, cuanto mayor vocabulario tenían los niños menos errores en la lectura y mayor destreza en el deletreo. Además, tanto la conciencia morfológica como la fonológica y el nivel de vocabulario correlacionaron 
significativamente entre sí de forma positiva. No obstante, los análisis de regresión realizados los llevaron a concluir que la contribución de la conciencia morfológica no iba más allá de la que aportaba la conciencia fonológica y el nivel de vocabulario. Este resultado estaría en contra de otros obtenidos en diversas lenguas en donde sí se ha comprobado la relación de la morfología con la destreza lectora y el deletreo. Para explicar esta divergencia se arguyeron dos posibles razones. La primera es que los resultados con lectores hábiles no tienen por qué representar lo que suceda en estos niños con dificultades lectoras. La segunda es que en español, lengua muy transparente desde el punto de vista fonológico, los resultados pueden diferir de otros como el inglés, en donde la opacidad fonológica es tal que podrían ponerse en juego otras estrategias para el desarrollo lector de los niños. Ambos argumentos vienen a reforzar lo que se adelantó más arriba, a saber, la casi total ausencia de estudios realizados en español con población con desarrollo típico limita nuestro entendimiento e interpretación de estos y otros resultados. En este contexto, el presente estudio se lleva a cabo con la intención de contribuir al conocimiento del papel que juega la conciencia morfológica en la habilidad lectora de niños hablantes nativos de español, en concreto pretendemos conocer si los distintos niveles de conciencia morfológica de los participantes se pueden relacionar con sus niveles de comprensión lectora y, además, si esta relación es independiente del efecto de otras variables relevantes como el nivel del vocabulario, la velocidad lectora, el CI o el nivel de conciencia fonológica. Más específicamente, planteamos la hipótesis de que los participantes con mayores niveles de conciencia morfológica presentarán una mayor comprensión lectora que los niños con menores niveles de la misma. Los resultados observados se referirán a la población con desarrollo típico hispanohablente de España, lo que complementará el mapa de resultados referidos a la relación entre conciencia morfológica y habilidad lectora observada en otras lenguas

\section{Método}

\subsection{Participantes}

Un total de 54 estudiantes (25 niñas y 29 niños) con edades comprendidas entre los 8 años y 1 mes y los 10 años y 3 meses participan en este estudio. De ellos, 24 (10 niños y 14 niñas) cursan $3^{\circ}$ de primaria $(M=8$ años y 9,5 meses; DT $=4$ meses) y 30 (15 niños y 15 niñas) cursan $4^{\circ}$ de primaria $(M=9$ años y 9 meses; DT $=4,5$ meses). Ambos grupos ( $3^{\circ}$ y $4^{\circ}$ de primaria) están equilibrados en cuanto al número de niños y niñas $y$, tanto los niños como las niñas, se distribuyen de forma equilibrada en ambos grupos, $\chi^{2}(1)=3,72 ; p=, 542$.

Se trata de una muestra incidental recogida en tres colegios de la Comunidad de Madrid. Para la realización del estudio los directores de los distintos colegios fueron informados y consintieron en que se contactara a los padres a través de los maestros para informarles del estudio. Solo aquellos niños que entregaron los consentimientos 
informados participaron en el estudio. El consentimiento informado se redactó de acuerdo con los estándares de la Declaración de Hensilki.

Todos los niños eran normolectores de acuerdo con los maestros tutores. No estaban siendo medicados ni asistían a apoyo logopédico ni psicológico. Como a continuación se detallará, se les administró el test K-Bit (Kaufman \& Kaufman, 1997) y las subpruebas de lectura de palabras y lectura de pseudopalabras del PROLEC-R (Cuetos, Rodríguez, Ruano \& Arribas, 2007) para verificar tanto que los participantes tenían unos niveles de inteligencia dentro de la normalidad como también unas habilidades lectoras normales.

\subsection{Materiales y procedimiento}

Se administraron distintas pruebas en un aula reservada en dos días distintos, con una duración total de una hora y cuarto. La administración de todas las tareas fue realizada por uno de los autores de este artículo. Las pruebas aplicadas fueron las siguientes:

Para evaluar la inteligencia se aplicó la prueba K-Bit (Kaufman \& Kaufman, 1997) que es un test breve de inteligencia, aplicable desde los 4 años hasta la edad adulta; mide inteligencia verbal y no verbal y proporciona una puntuación centil. Esta prueba presenta un coeficiente de fiabilidad alfa de Cronbach de 0,86.

Se aplicaron las subpruebas de lectura de palabras, lectura de pseudopalabras y de comprensión lectora del PROLEC-R (Cuetos et al., 2007). En todas ellas se puntúan los aciertos con un punto y las puntuaciones directas se transforman en categorías de normalidad, dificultad leve y dificultad severa. Esta prueba en su conjunto tiene un coeficiente de fiabilidad $\alpha=0,79$.

El nivel de vocabulario pasivo se midió mediante el test de vocabulario PeabodyPPVT III (Dunn, Dunn \& Arribas, 2011). Mide el nivel de vocabulario receptivo y puede servir de screening para la capacidad verbal. Se aplica a partir de los 2 años y medio hasta la edad adulta y proporciona puntuaciones centiles, CI, eneatipos, percentiles y edades equivalentes. Para las edades de los participantes en este estudio tiene unos valores de alfa de Cronbach entre 0,91 y 0,94.

La conciencia fonológica se midió con la subprueba de conciencia fonológica del CELF-4 (Semel, Secord \& Wiig, 2013). Es válida para niños entre 5 y 12 años y en ella se evalúa el conocimiento que los niños tienen de los fonemas y su habilidad para manipularlos, mediante tareas de: segmentación silábica y fonémica, unión de fonemas, supresión de sílabas y manipulación e identificación de fonemas. La fiabilidad de esta tarea estimada por el coeficiente alfa de Cronbach de esta tarea está entre 0,78 y 0,88 en las edades de los participantes de nuestro trabajo. 
La velocidad lectora se estimó mediante el número de palabras leídas correctamente en un minuto.

La conciencia morfológica se midió a través de un conjunto de tareas, tomadas de las utilizadas por distintos autores para medir este constructo y que serán detalladas a continuación. La fiabilidad de estas tareas fue determinada por el coeficiente alfa de Cronbach $(\alpha=0,77)$. Se calculó también la fiabilidad de las tareas experimentales para cada uno de los grupos de estudio: grupo de niños de $3^{\circ}, \alpha=0,83$; grupo de niños de $4^{\mathrm{o}}, \alpha=0,73$.

La primera tarea (T1) consiste en la elección de la definición correcta de una palabra polimorfémica leída por la evaluadora donde los participantes expresan oralmente cuál creen que es la definición correcta. Se emplean 10 estímulos en total.

Esta tarea se ha empleado también en las investigaciones de Carlisle y Fleming (2003).

Por ejemplo, 'Papelería':

1. Persona encargada de un establecimiento donde se vende papel y objetos de escritorio.

2. Establecimiento en el que se vende papel y otros objetos de escritorio.

3. Herramienta que se utiliza para cortar papeles.

La tarea 2 consiste en la definición de pseudopalabras. Por ejemplo se les pide que definan cristaloso. Esta tarea de definición ha sido empleada anteriormente de modo satisfactorio por autores como (Bertram, Laine \& Virkkala, 2000, Nicoladis \& Krott, 2007). Se emplean 10 estímulos en esta tarea.

La tarea 3 consiste en finalizar las oraciones derivando la palabra clave. Así por ejemplo se les enuncia la palabra 'actuar' y se les pide que finalicen la oración con una palabra derivada adecuada 'Esta noche es su última (actuación)'. Esta tarea se ha empleado previamente en trabajos como los de Carlisle (2000) o González et al. (2011) y constó de 17 estímulos.

La tarea 4 se centra en la prefijación y consiste en la generación de contrarios. Así se les da la palabra 'cómodo' y han de decir 'incómodo' o se les da 'posible' y han de decir 'imposible'. Autores como Contreras (2004) han empleado esta tarea. Se emplean un total de 10 estímulos.

La tarea 5 consiste en generar tantas palabras como se pueda de unas determinadas familias léxica. Así, por ejemplo, se da la palabra 'zapato' y se consideran palabras como 'zapatero', 'zapatería', etc. Esta tarea, que consta de ocho estímulos, ha sido adaptada y empleada en las investigaciones de Contreras (2004) o Vega et al. (2016). 
La tarea 6 se centra en la composición y consiste en completar oraciones. Así, un ejemplo de esta tarea sería 'Un niño que tiene el pelo rojo es'... o 'El electrodoméstico para limpiar la vajilla' es un... Esta tarea se ha utilizado en las investigaciones de García y Gonzalez (2006), López-Campelo, Fidalgo y García (2008). Esta tarea consta de nueve estímulos.

La tarea 7 es de naturaleza flexiva y consiste en detectar errores de conjugación nominal, ya sea de género o de número. Así, se dan a los niños expresiones correctas o incorrectas y deben indicar si están bien o no. Por ejemplo 'unas gatos', 'esta luces' o 'unos pantalones'. Este tipo de tarea ha sido utilizada anteriormente por Carlisle y Nomanbhoy (1993) o por González et al. (2011). En esta tarea se presentan 13 estímulos.

La tarea 8 es igualmente de naturaleza flexiva y consiste en conjugar el verbo correctamente de acuerdo al contexto. Así, se les dice 'ellas quedar mañana' y los niños deben decir 'ellos quedarán mañana'. Esta tarea fue utilizada por Contreras (2004) y constó de seis estímulos.

\subsection{Análisis de datos}

Para analizar la posible existencia de diferencias significativas en la competencia morfológica de los participantes en función del curso (tercero y cuarto), se llevó a cabo un análisis paramétrico, o su correspondiente no paramétrico, en función del cumplimiento, o no, de los supuestos requeridos. Para el estudio de las correlaciones en las puntuaciones de las distintas pruebas se ha aplicado la correlación de Pearson y, por último, previa comprobación de los supuestos, se llevó a cabo un análisis de regresión lineal múltiple por pasos para analizar la capacidad predictiva de las variables estudiadas sobre la comprensión lectora. Para estos análisis se ha empleado el programa IBM SPSS Statistics versión 22.

\section{Resultados}

Los estadísticos descriptivos (media y desviación típica) de las pruebas administradas a los participantes quedan recogidos en la tabla 1. La conciencia morfológica se operativizó en conciencia morfológica derivativa, obtenida como la suma de las puntuaciones de los participantes en las tareas 1 a 7 y en conciencia morfológica flexiva, suma en las tareas 7 y 8 . La conciencia morfológica derivativa y flexiva son las variables dependientes del estudio. 
Tabla 1. Estadísticos descriptivos en las pruebas realizadas por los participantes, en función del curso. Media (desviación típica).

\begin{tabular}{|c|c|c|c|c|c|c|c|c|c|}
\hline & \multirow{3}{*}{$\begin{array}{c}\text { Vocabulario } \\
\text { Peabody }\end{array}$} & \multirow{3}{*}{$\begin{array}{c}\text { Velocidad } \\
\text { lectora }\end{array}$} & \multicolumn{3}{|c|}{ Prolec-R } & \multirow{2}{*}{\begin{tabular}{|c|} 
Inteligencia \\
K-bit \\
\end{tabular}} & \multirow{2}{*}{$\begin{array}{c}\text { CELF-4 } \\
\text { Conciencia }\end{array}$} & \multirow{3}{*}{$\begin{array}{l}\text { Conciencia } \\
\text { morfológica } \\
\text { derivativa }\end{array}$} & \multirow{3}{*}{$\begin{array}{l}\text { Conciencia } \\
\text { morfológica } \\
\text { flexiva }\end{array}$} \\
\hline & & & Lectura & Lectura & Comprensión & & & & \\
\hline & & & Palabras & $\begin{array}{l}\text { Pseudo- } \\
\text { palabras }\end{array}$ & Textos & & Fonología & & \\
\hline \multirow{2}{*}{ Tercero } & 117,1 & 77,5 & 38,7 & 36,9 & 12,7 & 111,4 & 53,5 & 49 & 18,42 \\
\hline & $-14,45$ & $-25,87$ & $-1,46$ & $-2,29$ & $-2,57$ & $-14,17$ & $-2,32$ & $-9,48$ & $-0,77$ \\
\hline \multirow{2}{*}{ Cuarto } & 121,83 & 110,17 & 39,6 & 37,53 & 12,4 & 103,17 & 53,13 & 54,1 & 18,43 \\
\hline & $-12,91$ & $-27,31$ & $-0,77$ & $-1,8$ & $-1,83$ & $-9,15$ & $-1,94$ & $-7,85$ & $-0,77$ \\
\hline
\end{tabular}

Para la morfología derivativa, se llevó a cabo un ANOVA unifactorial con medidas independientes. Los resultados muestran un efecto significativo del curso $[\mathrm{F}(1,52)=$ 7,$32 ; p=, 04 ; \eta^{2}=, 077$; potencia $\left.=, 532\right]$, de forma que los alumnos de cuarto $(M=$ $72,53 ; D T=8,19)$ obtienen puntuaciones significativamente más altas que los de tercero $(M=67,42 ; D T=9,9)$.

Con respecto a la morfología flexiva, se llevó a cabo un análisis no paramétrico para muestras independientes mediante el test $U$ de Mann-Whitney. Los resultados señalan que no hay diferencias significativas, $[Z=-0,059 ; p=, 953]$, entre los alumnos de tercero (Rango promedio $=27,38$ ) y los alumnos de cuarto (Rango promedio $=$ 27,60). En esta variable, los alumnos de tercero y cuarto presentan medias muy similares (18,03 vs. 18,28) y muy próximas al límite superior (19 puntos). Los diagramas de caja (ver figuras 1 y 2) muestran un claro efecto techo para la morfología flexiva en ambos grupos y un mejor comportamiento para la morfología derivativa. 


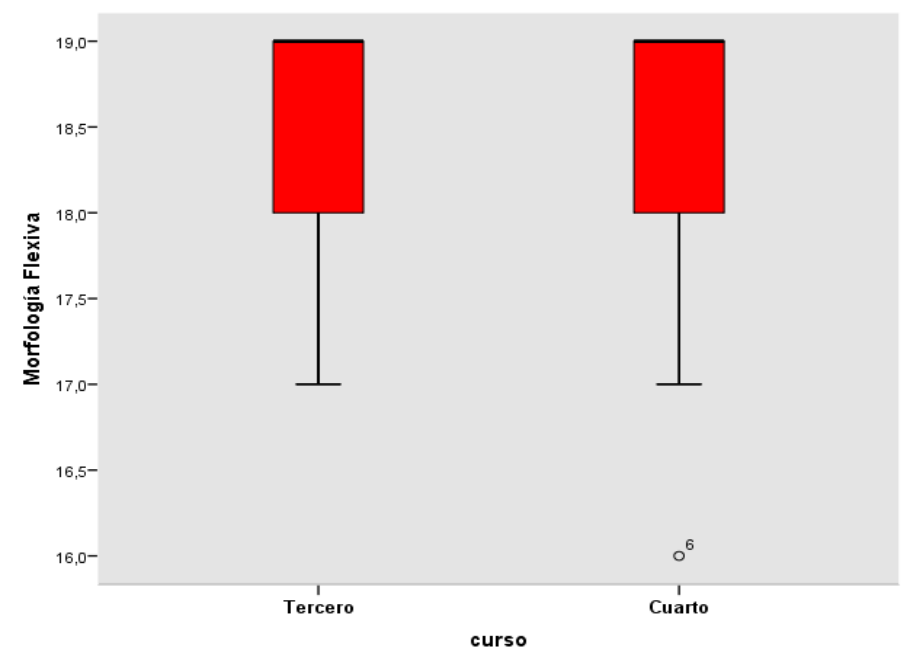

Figura 1. Diagrama de caja de la morfología flexiva en función del curso.

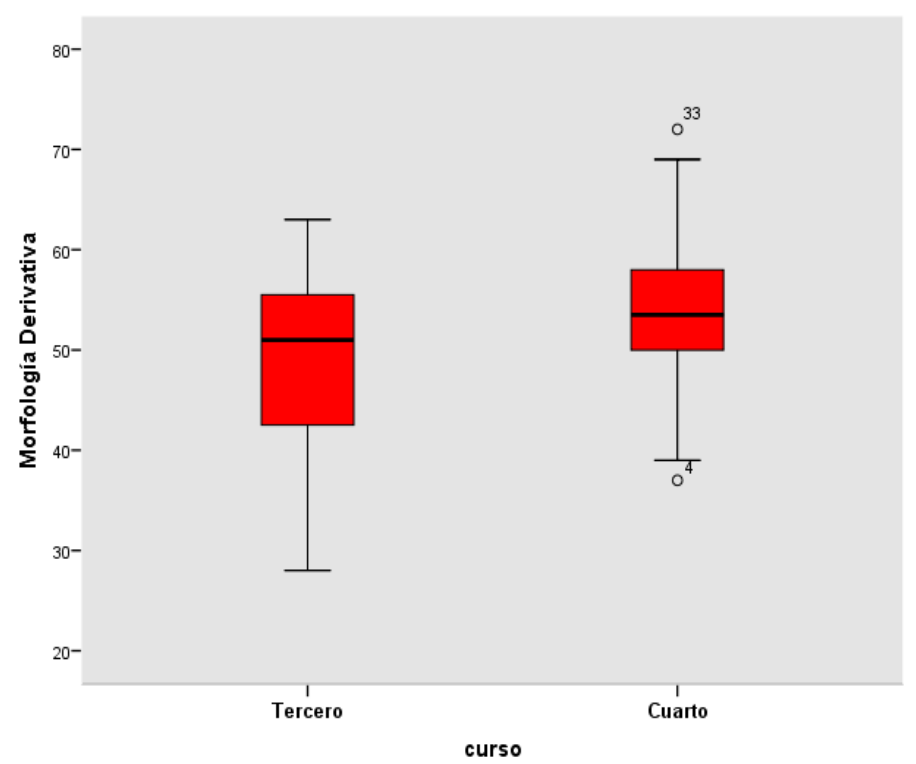

Figura 2. Diagrama de caja de la morfología derivativa en función del curso.

Respecto al análisis correlacional, considerando el efecto techo observado en las pruebas flexivas, se informa únicamente de los resultados de la morfología derivativa. Para cada uno de los cursos se obtuvo la correlación de Pearson entre esta variable y las puntuaciones obtenidas por los participantes en las distintas pruebas aplicadas. Con respecto a tercero, los resultados más relevantes muestran que la conciencia morfológica derivativa correlaciona positiva y significativamente con el nivel de vocabulario $(r=, 61 ; p=, 001)$, con la velocidad lectora $(r=, 43 ; p=, 034)$, con la 
comprensión de textos $(r=, 77 ; p<, 001)$, y con la conciencia fonológica $(r=, 43 ; p=$ ,038); ver Tabla 2 para los resultados completos.

Tabla 2. Correlación de Pearson entre las diferentes variables del estudio en el grupo de tercero de primaria.

\begin{tabular}{|l|c|c|c|c|c|}
\hline & $\begin{array}{c}\text { Conciencia } \\
\text { Morfológica } \\
\text { Derivativa }\end{array}$ & Vocabulario & $\begin{array}{c}\text { Velocidad } \\
\text { Lectora }\end{array}$ & $\begin{array}{c}\text { Comprensión } \\
\text { Textos }\end{array}$ & $\begin{array}{c}\text { Conciencia } \\
\text { Fonológica }\end{array}$ \\
\hline $\begin{array}{l}\text { Conciencia Morfológica } \\
\text { Derivativa }\end{array}$ & 1 &, $61^{* *}$ &, $43^{*}$ &, $77^{* *}$ &, $43^{*}$ \\
\hline Vocabulario & & 1 &, $37^{*}$ &, $72^{* *}$ &, $47^{*}$ \\
\hline Velocidad Lectora & & & 1 &, $39^{*}$ &, $38^{*}$ \\
\hline Comprensión Textos & & & & 1 &, $67^{* *}$ \\
\hline Conciencia Fonológica & & & & & 1 \\
\hline
\end{tabular}

Nota. ${ }^{*} \mathrm{p}<, 01 ;{ }^{*} \mathrm{p}<, 05$

Con respecto a cuarto de primaria, las correlaciones muestran que la conciencia morfológica derivativa correlaciona positiva y significativamente con el nivel de vocabulario $(r=, 50 ; p=, 005)$ y con la comprensión de textos $(r=, 45 ; p=, 013)$; ver Tabla 3 para los resultados completos.

Tabla 3. Correlación de Pearson entre las diferentes variables del estudio en el grupo de cuarto de primaria.

\begin{tabular}{|l|l|l|l|l|l|}
\hline & $\begin{array}{c}\text { Conciencia } \\
\text { Morfológica } \\
\text { Derivativa }\end{array}$ & Vocabulario & $\begin{array}{c}\text { Velocidad } \\
\text { Lectora }\end{array}$ & $\begin{array}{c}\text { Comprensión } \\
\text { Textos }\end{array}$ & $\begin{array}{l}\text { Conciencia } \\
\text { Fonológica }\end{array}$ \\
\hline $\begin{array}{l}\text { Conciencia Morfológica } \\
\text { Derivativa }\end{array}$ & 1 &, $50^{* *}$ & 0,25 &, $45^{*}$ & 0,28 \\
\hline Vocabulario & & 1 & 0,18 &, $58^{* *}$ &, $05^{*}$ \\
\hline Velocidad Lectora & & & 1 & 0,07 &, $46^{* *}$ \\
\hline Comprensión Textos & & & & 1 & $-0,14$ \\
\hline Conciencia Fonológica & & & & & 1 \\
\hline
\end{tabular}

Nota. ${ }^{* *} \mathrm{p}<, 01 ;{ }^{*} \mathrm{p}<, 05$

Por último, para cada uno de los cursos, se llevó a cabo un análisis de regresión lineal múltiple por pasos. La comprensión de textos fue la variable dependiente y el vocabulario, la conciencia fonológica y la conciencia morfológica derivativa fueron las variables predictoras.

En el caso de los alumnos de tercero de primaria, los resultados se muestran en la Tabla 4. Las variables finalmente incluidas en el modelo fueron la conciencia morfológica derivativa $(\beta=, 585)$ y la conciencia fonológica $(\beta=, 422)$, quedando excluido el vocabulario $(\beta=0,285 ; p=, 055)$. El porcentaje de varianza explicado por el modelo final es de un $70,5 \%$.

En la tabla 5 se muestran los resultados para los alumnos de cuarto curso. La única variable que finalmente queda en el modelo es el vocabulario $(\beta=, 543)$. En este caso, el porcentaje de varianza explicada es del 26,9\%. Han quedado excluidas del modelo la 
conciencia morfológica $(\beta=, 203 ; p=, 055)$ y la conciencia fonológica $(\beta=-, 170 ; p=$ ,293).

Tabla 4. Análisis de regresión lineal múltiple por pasos. Alumnos de tercero de primaria.

\begin{tabular}{|l|c|c|c|c|c|c|c|}
\hline & B & $\begin{array}{c}\text { Error } \\
\text { típico }\end{array}$ & $\boldsymbol{\beta}$ & $\boldsymbol{T}$ & $\boldsymbol{P}$ & $\begin{array}{c}\mathbf{R}^{2} \\
\text { corregida }\end{array}$ & $\begin{array}{c}\text { Sig. } \\
\text { cambio }\end{array}$ \\
\hline Modelo 1 & & & & & & & \\
\hline Constante) & 2,525 & 1,852 & & 1,364 & 0,187 & & \\
\hline Conciencia morfológica derivativa & 0,207 & 0,037 & 0,765 & 5,573 &, $000^{* *}$ & 0,566 &, $000^{* *}$ \\
\hline Modelo 2 & & & & & & & \\
\hline Constante) & $-20,033$ & 6,86 & & $-2,92$ &, $008^{* *}$ & & \\
\hline Conciencia morfológica derivativa & 0,158 & 0,034 & 0,585 & 4,676 &, $000^{* *}$ & & \\
\hline Conciencia fonológica & 0,466 & 0,138 & 0,422 & 3,373 &, $003^{* *}$ & 0,705 &, $003^{* *}$ \\
\hline
\end{tabular}

Note. Variable criterio: comprensión de textos; variables predictoras: vocabulario, conciencia fonológica y conciencia morfológica derivativa.

$* * \mathrm{p}<, 01 ; * \mathrm{p}<, 05$

Tabla 5. Análisis de regresión lineal múltiple por pasos. Alumnos de cuarto de primaria.

\begin{tabular}{|l|c|l|c|c|c|l|l|}
\hline & $\mathbf{B}$ & Error típico & $\boldsymbol{\beta}$ & $\boldsymbol{T}$ & $\boldsymbol{P}$ & $\mathbf{R}^{2}$ corregida & Sig. Cambio \\
\hline Modelo 1 & & & & & & & \\
\hline (Constante) & 3,029 & 2,757 & & 1,099 & 0,281 & & \\
\hline Vocabulario & 0,077 & 0,023 & 0,543 & 3,418 & 0,002 & 0,269 &, $002^{* *}$ \\
\hline
\end{tabular}

Note. Variable criterio: comprensión de textos; variables predictoras: vocabulario, conciencia fonológica y conciencia morfológica derivativa.

${ }^{*} \mathrm{p}<, 01 ;{ }^{*} \mathrm{p}<, 05$

\section{Discusión}

Los resultados obtenidos en esta investigación muestran, en primer lugar, que los niños de cuarto de primaria obtienen puntuaciones superiores a los de tercero en sus niveles de conciencia morfológica. Este resultado, siendo el esperado, avala la construcción del material expuesto pues refleja el patrón evolutivo de la conciencia morfológica derivativa en los cursos de tercero y cuarto de primaria tal y como se esperaba a la luz de las investigaciones previas. Estos materiales también han mostrado que, en estas mismas edades, no existe progresión en cuanto a las habilidades morfológicas flexivas. A nuestro juicio, este resultado implica, tal y como se defendió en la introducción del trabajo, que la conciencia morfológica flexiva tiene una evolución más temprana que la derivativa y que debe ser objeto de estudio de modo independiente a la derivativa. Aunque existen distintos, innumerables, modos posibles de evaluar la conciencia morfológica flexiva, hemos utilizado dos pruebas comunes en las investigaciones relacionadas, de modo que nuestros datos avalarían que la conciencia morfológica debe dividirse al menos en derivativa y flexiva y que la primera tiene una evolución más tardía (Tyler \& Nagy, 1989; Mahony et al., 2000; Deacon \& Bryant, 2005; González et al., 2011). A esta conclusión, basada en los datos 
obtenidos, añadimos lo expuesto en la introducción de este trabajo acerca de la distinta naturaleza de ambas morfologías, hecho conocido y tratado extensamente por la Lingüística, para justificar la propuesta de considerar en una única medida la conciencia morfología flexiva y la derivativa.

Por otro lado, los resultados de las correlaciones muestran resultados similares entre ambos cursos de los participantes, si bien existen también algunas diferencias. En cuanto a los resultados convergentes, tanto en tercero como en cuarto se ha observado una estrecha relación entre la conciencia morfológica derivativa y el nivel de vocabulario y, muy importante, con la comprensión de textos. Investigaciones previas ya habían relacionado la conciencia morfológica con el nivel de vocabulario (Ramírez, Walton \& Roberts, 2013). La relación en nuestro estudio entre los niveles de conciencia morfológica derivativa y el nivel de vocabulario es muy potente y tiene, desde el punto de vista teórico, fácil asiento una vez se expuso en la introducción la naturaleza precisamente léxica de la derivación. Como procedimiento de creación de palabras, la conciencia morfológica se relaciona naturalmente con el nivel de vocabulario pues mayores niveles de conciencia morfológica implican mayor capacidad para generar nuevas palabras y, por lo tanto, tener un amplio vocabulario (McBride-Chang et al., 2008; para una discusión en profundidad entre la relación entre vocabulario y conciencia morfológica).

Respecto de las variables de conciencia morfológica y comprensión de textos, los resultados avalan claramente la importancia del conocimiento morfológico puesto que se ha observado una relación significativa entre ambas variables por la que los participantes que mayores puntuaciones obtuvieron en las tareas de conciencia morfológica, mejor comprensión de textos demostraron. Estos datos sugieren que el conocimiento sobre las unidades morfológicas (lexemas y morfemas derivativos) ayuda a los lectores a construir los significados y sentidos de las oraciones y textos que leen, de modo que cuanto mayor sea el nivel de conciencia morfológica mayor será el beneficio que suponga a la comprensión de textos (Vega et al., 2016; Rueda \& Medina, 2018). Esta conclusión es coherente además con la correlación positiva y significativa entre nivel de vocabulario y la destreza lectora. Consideramos la conciencia morfológica como una habilidad muy estrechamente ligada al nivel de vocabulario, por lo que la naturaleza léxica de ambas variables justifica el que se encuentren resultados similares en su relación con la comprensión lectora. No obstante, no se trata de las mismas medidas ni mucho menos, ni en un sentido teórico ni en cuanto a los resultados obtenidos. A nivel teórico, la morfología derivativa, pese a su naturaleza léxica, también aporta información gramatical. Así, por ejemplo, un sufijo como -dor genera sustantivos o adjetivos que denotan el agente que realiza la acción del verbo (domador, vengador). La naturaleza léxica de la derivación es clara, pero su aporte gramatical implica una riqueza lingüística que explicaría adicionalmente que su mayor o menor dominio (operativizado a través de la conciencia morfológica) correlacione 
con las habilidades lectoras como la velocidad o, sobre todo, la comprensión de textos.

En el caso específico de tercero de primaria se ha observado también una estrecha relación entre conciencia morfológica y conciencia fonológica. La relación entre conciencia morfológica y fonológica había sido ya apuntada en diversas investigaciones (Kirby, Deacon, Bowers, Izenberg, Wade-Woolley \& Parrila, 2012; Law \& Ghesquière, 2017; Rueda \& Medina, 2018; Colé, Cavalli, Duncan, Theurel, Gentaz, Sprenger-Charolles et al., 2018). El papel de la conciencia fonológica en la lectura está hoy en día fuera de toda duda, así como poco a poco se está estableciendo para la conciencia morfológica, pero ¿por qué y cómo ambas conciencias se relacionan entre sí? Una posibilidad es que la habilidad en la conciencia fonológica ayude a segmentar morfológicamente las palabras. En este sentido, Casalis y Colé (2009) llevaron a cabo un programa muy completo de intervención tanto a nivel fonológico como morfológico controlando la habilidad lingüística a nivel oral. En sus resultados obtuvieron mejorías, pero en la interpretación de los datos propusieron precisamente que el incremento en la conciencia fonológica había ayudado a los niños a segmentar morfológicamente las palabras, de modo que su conciencia morfológica mejoró también. Igualmente el aumento en la conciencia morfológica había aumentado la sensibilidad a nivel fonológico de los participantes, de modo que la morfología había contribuido también al desarrollo fonológico. Se trataría por tanto de una relación bidireccional.

Siguiendo con esta relación entre fonología y morfología, en español, Lázaro, García y Burani (2015), realizaron dos experimentos en los que examinaron esta relación. En concreto en el primer experimento, en una tarea de definición de palabras polimorfémicas, observaron que aquellas palabras complejas en las que el sufijo casaba perfectamente con la base (como en futbol-ista) eran mejor definidas que aquellas otras en las que se producía una alteración morfo-fonológica (como en dientedentista). Estos resultados se observaron igualmente en una tarea de decisión léxica dado que las palabras sin alteración morfo-fonológica eran respondidas más rápidamente que aquellas otras en las que sí existían estas alteraciones. En las conclusiones, los autores indicaron que la segmentación morfológica requiere del desarrollo de las habilidades fonológicas que permitan a los lectores identificar los morfemas constitutivos de las palabras. Desde nuestro punto de vista, los resultados obtenidos en este estudio vienen a reflejar esta interrelación entre morfología y fonología por la cual ambas se influyen mutuamente. No obstante, los resultados de cuarto de primaria no muestran una correlación significativa entre ambas variables. ¿Por qué motivo no alcanza significación pese a la discusión teórica previa? Una posibilidad es que la destreza morfológica, significativamente superior en cuarto que en tercero, sea ya suficientemente elevada como para que sea más independiente y opere con menor influencia fonológica, es decir, que las habilidades morfológicas de 
los niños de cuarto estén ya suficientemente depuradas como para que su procesamiento sea menos sensible a la influencia fonológica. Si esto fuera así, en efecto, la relación entre ambas variables no tendría por qué ser significativa en términos estadísticos. Este resultado coincide con el previo de Deacon y Kirby (2004) donde se encontró que la conciencia morfológica explicaba el rendimiento en comprensión lectora durante un periodo más extenso que la conciencia fonológica, por lo que es posible que en edades inferiores ambas variables tengan un curso de desarrollo más relacionado que en cursos superiores. No obstante, esta posibilidad que apuntamos necesita de mayor soporte empírico, por lo que claramente es necesario profundizar en esta relación entre nivel de conciencia morfológica e influencia fonológica.

Los resultados de tercero también muestran una estrecha relación entre conciencia morfológica y velocidad lectora. Esta relación, novedosa en la bibliografía existente, viene a poner de relieve el papel de la morfología en una variable que es conocida por su relación con la habilidad lectora. Una posible explicación para esta relación puede tomarse del trabajo de Burani, Marcolini, de Luca, y Zoccolotti (2008), quienes destacan que la morfología derivativa constituye una unidad intermedia entre el reconocimiento de los grafemas y el de las palabras y que, por ello, el procesamiento de los morfemas supone un acceso más rápido a las palabras que un procesamiento más de tipo fonológico. Así, en niños en los que la destreza lectora está aún en desarrollo y el reconocimiento global de las palabras es una habilidad aún incipiente y poco eficaz, estas unidades mayores que los grafemas podrían ser de ayuda para incrementar la fluidez lectora (ver también Schmalz, Marinus \& Castles, 2013). Por lo tanto, podría interpretarse la relación significativa entre velocidad lectora y conciencia morfológica como evidencia de un uso eficaz de los morfemas en el acceso y reconocimiento léxico.

Con respecto a los análisis de regresión realizados, los resultados han mostrado en tercero de primaria que la conciencia morfológica, conjuntamente con la conciencia fonológica, explica un porcentaje significativo y muy alto de la varianza de la compresión de textos, en concreto, los datos muestran que es explicada el $70 \%$ de la varianza (56\% cuando se considera únicamente la conciencia morfológica). Este dato avala la relevancia de la conciencia morfológica, y no solo de la fonológica, en la comprensión de textos. No obstante, en cuarto de primaria, los resultados difieren: es el vocabulario la variable con mayor poder predictivo en el modelo de regresión; modelo que explica un porcentaje de varianza sensiblemente inferior $(26,9 \%)$ al explicado en el caso de tercero. La importancia del vocabulario en este caso va en la línea de lo que Rueda y Medina (2018) apuntan a la hora de destacar el papel del nivel del vocabulario incluso por encima del papel de la conciencia morfológica. No obstante en aquel trabajo los participantes habían sido diagnosticados con dificultades lectoras, lo que dificulta una comparación directa con nuestros datos. Lo que 
observamos en nuestros resultados es una correlación muy elevada entre conciencia morfológica y nivel de vocabulario. Aunque esta alta correlación se observa también en tercero, los datos evolutivos muestran mayor rendimiento en los participantes de cuarto, motivo por el que podría defenderse que el modo en que las distintas variables estudiadas interactúan entre sí para contribuir a la comprensión de textos podría tener una naturaleza muy interactiva y adaptativa a las habilidades específicas de los niños, por lo que, consiguientemente, podrían variar los resultados en función de los cursos estudiados. Esta conclusión implica la necesidad de profundizar en futuras investigaciones y recuerda la gran carestía de datos experimentales específicos del español, lo que dificulta perfilar una conclusión basada en datos y discusiones previas para nuestra lengua

A modo de conclusión podemos indicar que los resultados de este estudio ponen de relieve que:

- El curso temporal de la adquisición del conocimiento flexivo es anterior a la del derivativo. Esto, junto a la distinta naturaleza de ambos tipos de morfología, debe considerarse a la hora de establecer los niveles de conciencia morfológica.

- Los materiales desarrollados en este estudio para evaluar la conciencia morfológica han detectado una evolución en la misma en los niños de tercero y cuarto de primaria.

- La conciencia morfológica mantiene una estrecha relación con el nivel de vocabulario y con la comprensión lectora, lo que avalaría los programas de intervención morfológica en niños con dificultades lectoras (Carlisle, 2000; Verhoeven \& Perfetti, 2003; Carlisle \& Stone 2005; McBride-Chang et al., 2005; García \& González, 2006; Wolter, Wood \& D'Zatko, 2009; Traficante, 2018).

Son necesarias más investigaciones en lengua española para progresar en nuestro conocimiento del papel que desempeña la morfología en la habilidad lectora. En concreto, nuestros datos apuntan al interés de avanzar desde un punto de vista evolutivo en la interrelación entre las conciencias morfológica y fonológica y también entre el nivel de vocabulario y la conciencia morfológica.

\section{REFERENCIAS BIBLIOGRÁFICAS}

Apel, K. (2014). A Comprehensive definition of morphological awareness implications for assessment. Topics in Language Disorders, 34, 197-209.

Aronoff, M. \& Fudeman, K. (2005). What is morphology? Fundamental of Linguistics. Nueva Jersey: Wiley-Blackwell.

Berko, L. (1958). The child's learning of English morphology. Word, 14, 150-177. 
Bertram, R., Laine, M. \& Virkkala, M.M. (2000). The role of derivational morphology in vocabulary acquisition: Get by with little help from my morpheme friends. Scandinavian Journal of Psychology, 41, 287-296.

Booig, G. (2002). The morphology of Dutch. Oxford: Oxford University Press.

Burani, C., Marcolini, S., de Luca, M. \& Zoccolotti, P. (2008). Morpheme-based reading aloud: Evidence from dyslexic and skilled Italian readers. Cognition, 108, 243-262.

Carlisle, J. F. (2000). Awareness of the structure and meaning of morphologically complex words: Impact on reading. Reading and Writing, 12, 160-190.

Carlisle, J. F. \& Nomanbhoy, D. M. (1993). Phonological and morphological awareness in first graders. Applied Psycholinguistics, 14, 177-195.

Carlisle, J. F. \& Fleming, J. (2003) Lexical processing of morphologically complex words in the elementary years. Scientific Studies of Reading, 7, 239-253.

Carlisle, J. F. \& Stone, C. (2005). Exploring the role of morphemes in word reading. Reading Research Quarterly [en línea]. Disponible en: https://doi.org/10.1598/RRQ.40.4.3

Casalis, S. \& Colé, P. (2009). On the relationship between morphological and phonological awareness: Effect of training in kindergarten and in first-grade reading. First Language, 29, 113-142.

Colé, P., Cavalli, E., Duncan, L.G., Theurel, A., Gentaz, E., Sprenger-Charolles, L. et al. (2018). What is the influence of morphological knowledge in the early stages of reading acquisition among low SES children? A graphical modeling approach. Frontiers in Psychology, 9. DOI: 10.3389/fpsyg.2018.00547

Contreras, M. G. (2004). Habilidades morfológicas y experiencia socioeducativa. Arbor, 179, 213-227.

Cuetos, F., Rodríguez, B., Ruano, E. \& Arribas, D. (2007). Prolec-R: Batería de evaluación de los procesos lectores. Madrid: TEA Ediciones.

Deacon, S. H. \& Kirby, J. R. (2004). Morphological awareness: Just "more phonological"? The roles of morphological and phonological awareness in reading development. Applied Psycholinguistics, 25(2), 223-238.

Deacon, S. \& Bryant, P. (2005). What young children do and do not know about the spelling of inflections and derivations. Developmental Science, 8, 583-594.

Dunn, L. M., Dunn, L. \& Arribas, D. (2011). Peabody: Test de vocabulario en imágenes. Madrid: TEA Ediciones. 
García, J. \& González, L. (2006). Diferencias en la conciencia morfológica, la escritura y el lenguaje en función del desarrollo y el nivel educativo del niño. Psicothema, 18, 171-179.

González, L., Rodríguez, C., Gázquez, J. J., González. P. \& Álvarez, D. (2011). La conciencia morfológica: Tendencias de desarrollo y patrón evolutivo en Educación Infantil y Primaria. Psicothema, 23, 239-244.

Greenberg, J. (1963). Some universals of grammar with particular reference to the order of meaningful elements. En J. Greenberg, (Ed.), Universals of Language. (pp. 58-90). Massachusetts: The MIT Press.

Kaufman, A. \& Kaufman, N. (1997). K-bit: Test Breve de Inteligencia de Kaufman. Madrid: Pearson.

Kieffer, M. \& Lesaux, N. (2008). The role of derivational morphology in the reading comprehension of Spanish-speaking English language learners. Reading and Writing, 21, 783-804.

Kirby, J., Deacon, H., Bowers, P., Izenberg, L., Wade-Woolley, L. \& Parrila, R. (2012). Children's morphological awareness and reading ability. Reading and Writing, 25, 389-410.

Law, J. \& Ghesquière, P. (2017). Early development and predictors of morphological awareness: Disentangling the impact of decoding skills and phonological awareness. Research in Developmental Disabilities, 67, 47-59.

Lázaro, M., Nieva, S., Moraleda, E. \& Garayzábal, E. (2013). Procesamiento morfológico y formación del plural en niños con desarrollo típico. Revista de Didácticas Específicas, 8, 65-80.

Lázaro, M., García, C. \& Burani, C. (2015). How orthographic transparency modulates morphological processing in young readers with and without reading disability. Scandinavian Journal of Psychology. DOI: 10.1111/sjop.12213

López-Campelo, B., Fidalgo, R. \& García, J. (2008). La evaluación de la conciencia morfológica- PCM. Su papel en la adquisición de la lectura. International Journal of Developmental and Educational Psychology, 1, 425-434.

López-Ornat, S. (2011). La adquisición del lenguaje. Revista de Investigación en Logopedia, $1,1-11$.

Mahony, D., Singson, M. \& Mann, V. (2000). Reading ability and sensitivity to morphological relations. Reading and Writing, 12, 191-218. 
Mann, V. \& Singson, M. (2003). Linking morphological knowledge to English decoding ability Large effects of little suffixes. En E. Assinck \& D. Sandra (Eds.), Morphology and reading (pp. 1-25). Ámsterdam: Kluver Publishers.

Marrero, V. \& Aguirre, C. (2003). Plural acquisition and development in Spanish. En S. Montrul \& F. Ordóñez (Eds.), Linguistic theory and language development in hispanic languages: Papers from the 5th linguistics symposium and the 4th conference on the acquisition of Spanish and Portuguese (pp. 275-296). Somerville, MA: Cascadilla Press.

McBride-Chang, C., Wagner, R., Muse, A., Chow, B. \& Shu, H. (2005). The role of morphological awareness in children's vocabulary acquisition in English. Applied Psycholinguistics, 26, 415-435.

McBride-Chang, C., Tardif, T., Cho, J., Shu, H., Fletcher, P., Stokes, S., Wong, A. \& Leung, K. (2008). What's in a word? Morphological awareness and vocabulary knowledge in three languages. Applied Psycholinguistics, 29, 437-462.

Mínguez, C. M. (2018). Influencia de las conciencias fonológica y morfológica en la adquisición de la lectura. Estudios Interlingüísticos, 6, 96-115.

Moreno, J. C. (1997). Introducción a la lingüistica: Enfoque tipológico y universalista. Madrid. Síntesis.

Moreno, J. C. (2003). Síntesis y análisis en las lenguas: crítica de la tipología morfológica clásica y de algunas de sus aplicaciones sincrónicas y diacrónicas. Estudios de Lingüística, 17, 465-504.

Nicoladis, E. \& Krott, A. (2007). Word family size and French-speaking children's segmentation of existing compounds. Language Learning, 57, 201-228.

Pérez-Pereira, M. (1989). The acquisition of morphemes: Some evidence from Spanish. Journal of Psycholinguistics Research, 18, 289-312 [en línea]. Disponible en: https://doi.org/10.1007/BF01067038.

Ramírez, G., Walton, P. \& Roberts, W. (2013). Morphological awareness and vocabulary development among kindergarteners with different ability levels. Journal of Learning Disabilities, 47, 54-64.

Rey-Debove, J. (1984). Le domaine de la morphologie lexicale. Cabiers de Lexicologie, 45, 3-19.

Rueda, M. \& Medina, S. (2018). The role of morphological awareness in explaining reading-writing difficulties. Infancia y Aprendizaje, 41, 702-732. 
Schmalz, X., Marinus, E. \& Castles, A. (2013). Phonological decoding or direct access? Regularity effects in lexical decisions of Grade 3 and 4 children. The Quarterly Journal of Experimental Psychology, 66, 338-346.

Selvy, S. (1972). The development of morphological rules in children. British Journal of Educational Psychology, 42, 293-299.

Semel, E., Secord, W. \& Wiig, E. (2013). CELF-4: Spanish Clinical Evaluation of Language Fundamentals-4. Pearson.

Spencer, A. (1998). Morphoponological operations. En A. Spencer \& A. Zwicky (Eds.), The Handbook of Morphology. (pp.12 3-143). Massachussets: WileyBlackwell.

Stump, G. (1998). Inflection. En A. Spencer \& A. Zwicky (Eds.), The Handbook of Morphology. (pp. 12-43). Massachussets. Blackwell Publishers.

Traficante, D. (2018). From graphemes to morphemes: An alternative way to improve skills in children with dyslexia. Revista de Investigación en Logopedia, 2(2), 163-185.

Tyler, A. \& Nagy, W. (1989). The acquisition of English derivational morphology. Journal of Memory and Language, 28, 649-667.

Varela, S. (1993). La formación de palabras. Madrid. Taurus Universitaria.

Vega, Y., Torres, A. \& del Campo, M. (2016). Habilidades metamorfológicas y su incidencia en la comprensión lectora. Revista Signos. Estudios de Lingüística, 50(95), 453-471.

Verhoeven, L. \& Perfetti, C.A. (2003). The role of morphology in learning to read. Scientific Studies of Reading, 7, 209-217.

Wolter, J. A., Wood, A. \& D'Zatko, K. (2009). The influence of morphological awareness on first-grade children's literacy development. Language, Speech, and Hearing Services in the Schools, 40(3), 1-13. 